\title{
Décentralisation et gouvernance locale : " vers une incohérence du système municipal québécois "
}

\author{
Robert J. Gravel et Alexandre Huard \\ ÉNAP - Université du Québec
}

$\not$ u cours des dernières décennies, le système municipal québécois a subi plusieurs réformes. La dernière a été la formation d'une structure permettant les défusions, alors que le gouvernement du Québec a permis la concrétisation des réactions d'une partie de la population qui exprimait son mécontentement face à la réorganisation municipale et aux fusions dites forcées.

À la suite des diverses réformes, il est nécessaire de se demander si l'ensemble du système municipal répond à une certaine logique et s'il est cohérent. Est-il possible de cerner des tendances à long terme, une vision claire de ce que sont et ce que deviennent les gouvernements locaux au Québec ? Comme hypothèse de travail, il est plausible de penser que les défusions viennent plutôt créer une incongruité dans le paysage déjà complexe du système municipal québécois.

C’est donc par le biais des concepts associés à la décentralisation et à la gouvernance locale que nous tentons de saisir la signification des réformes municipales au Québec, et en particulier des défusions et du processus de reconstitution des municipalités qui s’ensuit. Quelle place

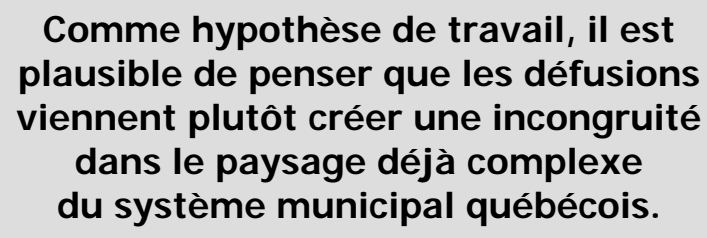

fusions. Elle se complexifie encore davantage lorsque celles-ci sont mises en relation avec les institutions régionales existantes, soit les anciennes Communautés urbaines (CU) maintenant agrandies et élargies en Communauté métropolitaine (CM) et les anciens Conseils régionaux de développement (CDR) transformés en Conférences régionales des élus (CRE). Ajoutons à cela la mise en place, là où il y a défusion, d'un Conseil d'agglomération (CA).

Ces organisations supramunicipales ont pour but d'effectuer une gestion d'agglomération de certains services. La création des CU avait pour but de répondre à des problématiques particulières. On cherchait à résoudre les problèmes liés au transport en commun, à la sécurité publique, à l'aménagement du territoire et ainsi de suite. Plus généralement, ces organismes tendaient à pallier à la parcellisation des municipalités et au besoin d'une gestion d'agglomération. Pour les MRC, leurs compétences étaient principalement limitées aux questions d’aménagement du territoire.

Les MRC sont de même nature que les communautés urbaines et elles regroupent surtout des municipalités qui ne sont pas de grands centres urbains. Néanmoins, on retrouve des MRC où une municipalité de grande taille prédomine sur des municipalités plus petites et à caractère rural. Les MRC regroupent ainsi des municipalités d'horizons divers ayant des besoins et intérêts parfois contradictoires, comme le sont les besoins urbains et les besoins ruraux. Cependant, les MRC sont très utiles comme forum d'ententes intermunicipales. De plus, les MRC sont souvent l'instance intermédiaire entre les municipalités et le gouvernement en étant celles qui représentent les intérêts des municipalités auprès du gouvernement.
La configuration complexe des pouvoirs qu'a instituée la réorganisation municipale concernait surtout les regroupements municipaux, et par la suite les dé-

\section{Les défusions et les instances régionales}


Les municipalités ont toujours été réticentes à une participation de la gestion intégrée d'une région. Ces réticences à la participation à cette gestion régionale sont les mêmes que celles qui ont mené à la défusion de certaines municipalités. Dès lors, il est possible d'avancer que la création d'instances régionales telles les CU et les MRC n'a pas permis de forger une volonté empressée de la part des instances locales à prendre pleinement part au développement régional.

Lorsqu'on met en relation cette réalité, ce manque de volonté de participer au niveau régional avec le processus des défusions, on découvre qu'il y a deux tendances de fond qui s'affrontent dans la configuration du système municipal québécois. 1) Le gouvernement du Québec, par ses réformes, semble adopter un modèle qui privilégie la gestion et le développement local dans une perspective régionale ou métropolitaine. Ainsi, par la création des instances supramunicipales et plus tard par les regroupements municipaux forcés, le gouvernement exprime sa volonté d'établir une configuration du système municipal qui répond davantage aux problématiques de développement régional qu'au développement purement local. 2) Toutes ces réformes n’ont pas amoindri le désir d'autonomie des municipalités qui expriment l'idée que seules les instances qui sont le plus près des citoyens répondent vraiment à leurs besoins. Le gouvernement du Québec, en permettant les défusions, vient donc ouvrir une brèche dans sa propre logique concernant le système municipal. Les défusions sont, entre autres, l'expression du refus de participer activement au développement régional. Ce refus et l'affirmation de la municipalité comme étant la seule instance locale légitime pour dispenser les services locaux se perpétue avec le combat contre les fusions municipales inaugurées par la ministre aux Affaires municipales du Québec, Madame Louise Harel.

Les défusions vont totalement à l'encontre des valeurs et des logiques qui sous-tendaient les autres réformes municipales qui visaient à développer une vision régionale de l'agglomération urbaine et de sa gestion.

\section{Les fusions et les défusions}

Les fusions municipales cherchaient à intégrer le développement économique, à transformer les questions de l'aménagement du territoire en préoccupations régionales, et non plus locales, et visaient à étendre le pouvoir des municipalités sur un plus vaste territoire. Jusqu'à un certain point, les regroupements municipaux venaient compléter l'élan d'une gestion régionale amorcée par la création des CU et des MRC. Certains ont soutenu que les villes feraient des économies d'échelle et que le déséquilibre entre la ville-centre et les municipalités périphériques serait enfin comblé. Aussi, pour d'autres, les fusions étaient le seul moyen viable pour offrir davantage d'autonomie aux instances locales.

C’est donc à partir de ces avancés que les défusions viennent semer la confusion au sein du paysage municipal du Québec. Ainsi, les défusions vont totalement à l'encontre des valeurs et des logiques qui soustendaient les autres réformes municipales qui visaient à développer une vision régionale de l'agglomération urbaine et de sa gestion. Les défusions sont le retour au modèle local où les décisions prises le plus près des citoyens seraient les meilleures décisions. Il y a une divergence profonde entre les visées des fusions municipales et des défusions. Mais les défusions viennent également à l'encontre des précédentes réformes du système municipal du Québec. Ainsi, le gouvernement du Québec a lui même créé une incohérence au sein de la lente progression du système municipal vers un système davantage orienté vers des préoccupations d’ordre régional.

Malgré tout, le nombre des municipalités qui ont défusionné est relativement bas. Ainsi, l'impact des défusionnistes ne sera peut-être pas très important sur la configuration du système municipal québécois, sauf à Montréal, Longueuil et, dans une moindre mesure, Québec. Néanmoins, notons que la divergence entre les partisans d'une plus grande autonomie locale et ceux qui favorisent la gestion régionale du territoire demeure. Et cette opposition est d'autant plus importante qu'elle a été institutionnalisée au sein même du système municipal québécois.

Ceci peut s'expliquer en partie par la loi adoptée par le gouvernement libéral permettant de démarrer le processus de défusion. Le projet de loi $n^{\circ} 9$ présenté en 2003 est un mélange de promesses électorales et de recul par rapport à la position du parti précédemment au pouvoir quant à la réorganisation municipale. C'est ainsi que, ne voulant pas laisser tomber une bonne partie de la population québécoise qui n’a pas appré- 
cié comment se sont déroulées la réorganisation municipale et les fusions forcées, le gouvernement à mis sur pied une politique mitoyenne puisqu'il ne voulait pas non plus revenir complètement sur les acquis des regroupements municipaux. C'est par ce processus que s’est forgée l'incongruité des défusions. Car en plus de ne pas correspondre aux buts des autres réformes municipales, la politique des défusions ne permet pas aux municipalités qui choisissent librement et légitimement cette option de retrouver l'intégralité de leur ville d'avant les fusions municipales.

C'est donc une double incohérence que viennent instituer les défusions. La première se pose par rapport aux autres réformes municipales, et la deuxième est la formation de municipalités de seconde zone. Comment distingue-t-on les arrondissements des villes fusionnées des villes nouvelles reconstituées ? Les municipalités reconstituées ont perdu beaucoup de leurs compétences. Elles se trouvent totalement dépendantes de la ville-centre au plan de certaines décisions majeures. Il est donc pertinent de mettre en comparaison les arrondissements des villes fusionnées avec les nouvelles municipalités reconstituées qui ont retrouvé leur autonomie en janvier 2006.

Les arrondissements des villes fusionnées ont d'abord un rôle consultatif. Ils présentent devant le Conseil de ville des propositions et des avis qu'ils jugent pertinents pour leur arrondissement. Mais ceux-ci possèdent aussi des compétences. La Charte de la ville de Québec ${ }^{1}$ mentionne, à l'article 114 , que les arrondissements ont des compétences en matière d'urbanisme, de sécurité incendie et de sécurité civile, d'enlèvement des matières résiduelles, de développement économique local, communautaire, culturel et social. Ils sont aussi responsables de la culture, des loisirs, des parcs au sein de leur territoire et de la voirie locale. Le cas de Montréal est spécifique. Il ne s'agit plus de président d'arrondissement mais bien d'un maire d'arrondissement. Cela a pour effet, comme le spécifie l'article 20 de la Charte de la ville de Montréal $^{2}$ que « le maire de l'arrondissement a, relativement aux compétences du conseil d'arrondissement, les pouvoirs, droits et obligations que la Loi sur les cités et villes (chapitre C-19) ou toute autre loi attribue au maire d'une municipalité locale ». Ainsi, une plus grande importance semble avoir été donnée aux arrondissements de Montréal par rapport à ceux de la ville de Québec.
On ne peut pas dire qu'il y aura une grande différence entre les arrondissements et les villes reconstituées, surtout si l'on compare celles-ci aux arrondissements de Montréal. En fait les projets de loi $n^{\circ} 9$ et $n^{\circ} 75$ qui légifèrent spécifiquement sur les questions des compétences portent davantage une attention particulière sur les compétences des agglomérations et les modalités de leur exercice que sur les compétences des municipalités qui seront reconstituées. N’oublions pas que les arrondissements ont la compétence en matière de développement économique local. Les villes reconstituées ont à peu près la même marge de manœuvre en matière de développement économique que les arrondissements puisqu'il s'agit d'une compétence exercée par l'agglomération. Ainsi, plusieurs points cruciaux de l'organisation d'une municipalité se retrouvent aux mains de la ville fusionnée. C'est donc parfois près de $50 \%$ d'un budget d'une municipalité reconstituée qui va directement dans les coffres de la ville fusionnée.

Le Conseil d’agglomération (CA), organe décisionnel formé avec le projet de loi $n^{\circ} 9$, regroupant la ville fusionnée et les municipalités reconstituées, celles-ci n'ont presque pas de poids devant la grande ville. En effet, au CA de Montréal, 16 municipalités (Montréal et les 15 villes reconstituées) sont représentées. Or, le maire de Montréal a $87 \%$ des voix, ce qui est proportionnel à la taille de sa population ${ }^{3}$. À Québec, la ville de Québec ${ }^{4}$ a un poids de $96 \%$, alors que l'AncienneLorette et Saint-Augustin-de-Desmaures ont ensemble un poids de $4 \%$. Ainsi, les défusions ne redonnent pas aux citoyens et aux élus les municipalités dans lesquelles ils vivaient avant les regroupements forcés.

\section{On ne peut pas dire qu'il y aura une grande différence entre les arrondissements et les villes reconstituées, surtout si l'on compare celles-ci aux arrondissements de Montréal.}

L’ensemble des éléments discutés jusqu'ici semble démontrer que les défusions sont une option qui vient accentuer l'incohérence du système municipal québécois. En fait, les défusions instaurent un double discours et une contradiction au sein du système municipal. Un double discours puisque les citoyens, en se positionnant par rapport aux regroupements municipaux, n’ont pas pu obtenir ce pourquoi ils avaient 
voté. Les défusions instaurent et institutionnalisent une contradiction au sein du système municipal puisqu'on y retrouve une volonté de configurer ce système de manière à ce qu'il réponde aux problématiques régionales et métropolitaines en même temps qu'un discours misant sur l'autonomie des municipalités.

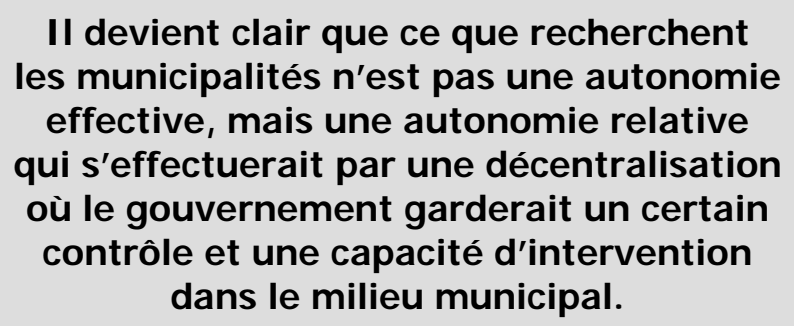

\section{Complication du système municipal québécois}

Les défusions municipales viennent complexifier le portrait du pouvoir local au Québec. Cette réalité complexe qu'est le système municipal québécois se laisse difficilement conceptualiser derrière des vocables comme la décentralisation ou le développement régional. Reliées à la gestion des municipalités (la décentralisation et la gouvernance locale) et la réalité du système municipal québécois, les défusions provoquent une rupture par rapport aux autres réformes municipales. Dans une perspective de décentralisation et de gouvernance locale, les municipalités qui ont été reconstituées le $1^{\mathrm{er}}$ janvier 2006 ne sont que l'ombre de ce qu'elles étaient avant le processus des fusions municipales. Leur souveraineté en matière de développement économique leur a été retranchée et elles doivent s'en remettre à la grande ville qui a le contrôle des décisions en matière de services d'agglomération. Ainsi, on peut noter que, loin de retrouver leur autonomie, les municipalités qui ont défusionné sont déchirées entre leur capacité d'établir des politiques locales adaptées à leurs besoins et leur dépendance face aux logiques de développement régional.

\section{La décentralisation}

La décentralisation est un concept qui est beaucoup discuté en matière de gestion municipale et métropolitaine. Par contre, cette notion est souvent mal comprise et interprétée. Ainsi, le contexte historique du développement des instances locales n'a pas favo- risé l'autonomie de celles-ci. Cependant, la réforme de la fiscalité a accru l'autonomie fiscale et financière des municipalités, même si des réformes plus récentes ont plutôt semblé valoriser une multiplication des instances locales et régionales qu'une augmentation de l'autonomie des municipalités. Celles-ci n’ont pas une mainmise parfaite sur leurs décisions puisqu'elles doivent toujours s'arrimer aux paliers régionaux et supramunicipaux de décision comme les MRC, les CM, les CRÉ ou les CA.

Le plus important quant à la notion de décentralisation est l'établissement d'une gouvernance locale pour le système municipal du Québec qui est peut-être la prépondérance que prend le gouvernement provincial dans le façonnement de ce que doivent être les villes et les municipalités. Plusieurs décisions du gouvernement ont démontré son contrôle sur le système municipal du Québec. Par exemple, la réforme Ryan de 1991 a été qualifiée de «diktat » par l'UMQ ${ }^{5}$. Aussi, la réforme Harel qui forçait les regroupements municipaux a démontré l'ampleur de la mainmise du gouvernement sur les municipalités. Jacques Léveillée $e^{6}$, de l'UQAM, mentionne qu'en dépit du discours qu'on tient sur la capacité des élus locaux à articuler les véritables besoins des collectivités locales, entre autres au plan des structures politiques et administratives de fonctionnement, ce sont les hommes politiques et les hauts fonctionnaires actifs au niveau central qui se reconnaissent la responsabilité, et se donnent le mandat, de proposer, voire d'imposer les nouveaux arrangements politico-administratifs qui devraient régir le fonctionnement des gouvernements locaux ainsi que les rapports entre les instances locales, les instances intermédiaires et les instances centrales de l'appareil étatique.

En fait, il est bien difficile, voire totalement incongru, de parler de décentralisation des municipalités au Québec lorsque, manifestement, celles-ci sont impliquées dans une relation d'ordre hiérarchique avec le gouvernement. Aussi, il faut comprendre que les municipalités du Québec ne recherchent peut-être pas la décentralisation parfaite. L'UMQ reconnaît le rôle majeur que tient le gouvernement dans l'évolution et le développement des villes et des municipalités. Elle souhaite même voir le gouvernement prendre davantage position dans les affaires municipales. Par exemple, l’UMQ mentionne que « la réussite de tout projet de décentralisation [...] dépend à coup sûr du partenariat véritable qui doit être mis sur pied entre le gou- 
vernement et le monde municipal $»^{7}$. Et aussi « [...] l'UMQ réitère son désir de voir le gouvernement jouer un rôle prépondérant à l'avenir en matière économique et social ${ }^{8}$. Il devient clair que ce que recherchent les municipalités n'est pas une autonomie effective, mais une autonomie relative qui s'effectuerait par une décentralisation où le gouvernement garderait un certain contrôle et une capacité d'intervention dans le milieu municipal.

Les défusions et leurs partisans viennent instaurer une rupture face à ce modèle. On peut interpréter les défusions comme étant une manifestation d'un profond désaccord de la part des élus et des citoyens par rapport à cette dépendance du monde municipal face au gouvernement du Québec. Les défusionnistes seraient alors des tenants d'une décentralisation qui laisse davantage une autonomie locale aux municipalités. À ceux-ci, plusieurs pourraient répondre que les instances locales, telles qu'elles étaient avant les fusions, n'étaient pas aptes à soutenir une plus grande autonomie et que les regroupements municipaux visaient justement la décentralisation du système municipal. Il reste quand même que les défusions proposent une autre vision de ce que devrait être la configuration des pouvoirs locaux par rapport au gouvernement.

Néanmoins, les villes reconstituées sont encore parties prenantes du jeu de dépendance qu'instaurent les différents paliers décisionnels au sein de l'organisation locale et régionale. Au lieu de retrouver plus d'autonomie, les villes reconstituées sont sous l'emprise d'un grand contrôle. Ainsi, en voulant exprimer leur autonomie et en voulant la sauvegarder, les villes qui ont défusionné se retrouvent dans la situation inverse où leur autonomie d'action devient très limitée. Par exemple, une ville comme Saint-Augustin dans la région de Québec n’a plus le contrôle de son développement économique puisque cela relève dorénavant de la gestion du Conseil d'agglomération (CA) de Québec.

\section{La gouvernance urbaine}

La gouvernance fait souvent référence à moins de gouvernement pour une meilleure gestion. La gouvernance est davantage perçue comme étant une intégration de divers paliers décisionnels et de différents acteurs pour la création de politiques publiques cohérentes qui répondent à des problématiques d'ordre régional ou métropolitaine.
L'analyse du système municipal et supramunicipal après les réformes du gouvernement du Québec semble démontrer que les intentions du gouvernement étaient de créer une dynamique régionale où la gouvernance serait le cœur de cette même dynamique. Ainsi, la création des CU et des MRC, les regroupements municipaux ainsi que la formation des CM et des CRÉ s'engageaient dans la voie de la gouvernance locale. Dès lors, on sort des chemins de la décentralisation qui confère davantage d'autonomie aux instances locales et ainsi on se tourne plutôt vers une interdépendance des différents paliers décisionnels locaux et régionaux qui donnent l'aspect d'une recentralisation.

\section{Après les défusions, les refusions ?}

Retournement inattendu et quelque peu farfelu, un discours sur la refusion de quelques municipalités défusionnées est en train d'émerger sur la scène politique au Québec. En fait, plus qu'un discours, la refusion a fait l'objet d'un référendum à Cap-auxMeules, municipalité défusionnée des Îles-de-laMadeleine. Les citoyens ont voté pour un renversement de la décision qu'ils avaient prise eux-mêmes lors du référendum du 20 juin 2004 sur les défusions.

Cette situation est très inconfortable pour l'actuelle ministre des Affaires municipales et des Régions, Madame Nathalie Normandeau. Le référendum qui a eu lieu à Cap-aux-Meules n'avait d'autre valeur que consultative. Si la ministre avalise le résultat de ce référendum, plusieurs autres municipalités comme Ancienne-Lorette pourraient se prévaloir du droit de refusion. Cependant, « il serait inutile de tenir un référendum sur la refusion de L'Ancienne-Lorette, comme le demande le conseiller Daniel Dupuis, parce que le gouvernement provincial n'en tiendra pas compte », avertit la députée de La Peltrie, France Hamel $^{9}$. Néanmoins, le phénomène de refusion vient jeter un peu de discrédit sur la politique des défusions telle qu’élaborée par le Parti libéral du Québec.

Les citoyens n'acceptent plus la solution mitoyenne qui leur est proposée, d'autant que le comité de transition vient d'annoncer que les coûts de défusion seront une augmentation de la taxation de $11 \%$ (soit 196 \$ : maison moyenne) pour la municipalité de l'Ancienne-Lorette et de $25 \%$ (soit 538 \$: maison moyenne) pour la municipalité de Saint-Augustin-deDesmaures ${ }^{10}$ pour avoir le même niveau de services qu'en 2005. 
À Montréal, les hausses d'impôt foncier varieront du statu quo à plus de 1000 \$ pour 2006. À titre d'exemple, pour une habitation unifamiliale, l'impôt foncier coûtera 1004 \$ de plus à Westmount, 500 \$ à Montréal-Ouest, 649 \$ à Beaconsfield, 1132 \$ à Senneville et 527 \$ à Dollard-des Ormeaux ${ }^{11}$.

Cette situation est également symptomatique du manque de cohérence qu'il y a dans le système municipal au Québec. Les défusions ont ouvert la porte à des politiques qui ne cadrent pas dans la logique des réformes qui ont précédé les défusions. Bien que le phénomène de refusion soit marginal, il dénote le malaise d'une partie des citoyens face aux défusions. Celles-ci n'ont pas offert aux citoyens ce qu'ils attendaient d'un processus clair laissant la décision au vote démocratique. Les refusions ne devraient pas être la norme. Ce sont plutôt dans des luttes pour obtenir davantage de pouvoir que les municipalités reconstituées s'engageront. Celles-ci chercheront à retrouver l'intégralité de leur municipalité. N'oublions pas que près de $50 \%$ des budgets des municipalités qui ont défusionné iront dans les coffres de la grande ville. Ainsi, on peut prévoir que les municipalités mettront une forte pression sur le gouvernement du Québec.

Pour l'instant, les municipalités qui ont opté pour la défusion suivent le processus qui les mènera à leur reconstitution. À l'automne 2005, il y eu des élections et les citoyens ont choisi leur conseil de ville. La pression sur le gouvernement devrait augmenter lorsque les élus auront retrouvé le cadre limité de leur municipalité. Par exemple, dans la région de Québec, la municipalité de Saint-Augustin tentera de remettre la main sur son parc industriel.

\section{Un paysage municipal chaotique}

C'est justement une juxtaposition de logiques contradictoires au sein même du système municipal québécois qui le met en butte aux incongruités et aux incohérences. Principalement, et c'est ce qui ressort de cette analyse, les défusions ont inséré un autre modèle de configuration des diverses instances décisionnelles locales et régionales que celui qui prédominait jusqu'à maintenant. Les réformes avant les défusions convergeaient toutes vers le passage d'une gestion fragmentée et parcellaire du territoire à une gestion intégrée présentant des politiques cohérentes au niveau métropolitain et régional.
La tendance à l'autonomie a toujours existé dans les organisations politiques locales. Ce désir d'autonomie est tout à fait légitime. Seulement, il ne correspond pas au modèle municipal que le gouvernement du Québec voudrait construire. Celui-ci a toujours eu la mainmise sur les municipalités, et toutes les réformes qui ont émané du gouvernement tendaient vers la planification et le développement régional. Les défusions instaurent une rupture dans le système. Le gouvernement a lui-même ouvert une brèche dans sa propre politique. Il a institutionnalisé à l'intérieur des structures de pouvoir locales et régionales l'opposition entre les tenants de l'autonomie locale et ceux davantage préoccupés par les problématiques régionales. Cette opposition n'est pas nouvelle. Ce qui est nouveau, c'est que, par la politique des défusions, le gouvernement a implanté ce bouleversement en instaurant une confusion et une complication menant à une incohérence de la gouvernance municipale au Québec.

\section{Notes et références}

1 Voir le site :

http ://www.ville.quebec.qc.ca/fr/organisation/ docs/charte_ville_quebec.pdf (p. 40).

2 Consulter :

http ://www2.publicationsduquebec.gouv.qc.ca/ dynamicSearch/telecharge.php ?type=2\&file=/C_11_4/C1 1_4.html

$3 \quad$ Le Devoir, 12 novembre 2004, p. A1.

4 Radio-Canada, 6 octobre 2005.

5 UMQ (1994), La décentralisation et les municipalités : les enjeux, le potentiel, les étapes préalables à une réalisation efficiente, Sherbrooke, Union des municipalités du Québec, p. 13.

6 Léveillée, J. (1979), Réorganisation de l'appareil d'État et réforme institutionnelle locale: une illustration. L'expérience québécoise, Québec, École nationale d'administration publique, p. 3.

7 UMQ (1994), op. cit., p. 103.

8 Idem, p. 93

9 Le Soleil, 24 avril 2005, p. A9.

10 Le Soleil, 5 octobre 2005, p. A1.

11 La Presse, 5 octobre 2005, p. A11. 